\title{
Overall Spectral Properties of Prompt Emissions with Diverse Segments in Swift/BAT Short Gamma-ray Bursts
}

\begin{abstract}
X.J. Li ${ }^{1}$, Z.B. Zhang*1, and K. Zhang ${ }^{1}$
School of Physics and Physical Engineering, Qufu Normal University, Qufu 273165, China

e-mail: astrophy0817@163.com

Received 2021 March 8; Accepted 2021 October

\section{ABSTRACT}

Owing to lack of multiple components of prompt $\gamma$-ray emissions in short gamma-ray bursts (sGRBs), how these distinct components are correlated still keeps unclear. In this paper, we investigate the spectral and temporal properties of precursors, main peaks and extended emissions in 26 sGRBs including GRB 170817A. It is found that peak energies $\left(E_{p}\right)$ in each pulse are uncorrelated with the pulse duration $\left(t_{d u r}\right)$. Meanwhile, we find that there is no obvious correlation between peak energy and energy fluence. Interestingly, there is no obvious spectral evolution from earlier precursors to later extended emissions in view of the correlations of $t_{d u r}$ with either the $E_{p}$ or the low energy spectrum index, $\alpha$. A power-law correlation between the average flux $\left(F_{p}\right)$ and the energy fluence $\left(S_{\gamma}\right)$, $\log F_{p}=(0.62 \pm 0.07) \log S_{\gamma}+(0.27 \pm 0.07)$, is found to exist in the individual segments instead of mean peaks previously. Furthermore, we also find that the main peaks are on average brighter than the precursors or the extend emissions about one order of magnitude. On the basis of all the above analyses, one can conclude that three emissive components would share the same radiation mechanisms but they might be dominated by diverse physical processes.
\end{abstract}

Key words. Stars: late-type - Gamma-ray burst: general - Radiation mechanisms: general - Methods: statistical

\section{Introduction}

The Swift satellite was successfully launched in 2004 November (Gehrels et al. 2004) and has detected over 1300 gamma-ray bursts (GRBs) till 2019 November. According to the classification criterion of the $T_{90}$ duration distribution (Kouveliotou et al. 1993), approximately $10 \%$ are short GRBs (sGRBs), with a typical duration of $T_{90}<2$ s (Zhang \& Choi 2008; Zhang et al. 2018). Prompt garmma-ray emissions of GRBs may consist of diverse components, namely precursors, main peaks and extended emissions (EEs), or parts of them, within both long GRBs (1GRBs) and sGRBs (Metzger et al. 1974; Koshut et al. 1995; Norris \& Bonnell 2006; Troia et al. 2010; Bernardini et al. 2013; Hu et al. 2014; Lan et al. 2018, 2020; Zhang et al. 2018b; Zhong et al. 2019; Zhang et al. 2020a; Li et al. 2021). The precursor reported first in GRB 720427 is a dim peak occurring before the brightest prompt emission of main peaks (Metzger et al. 1974), and the EE as the softer $\gamma$-ray emissions usually following the main peaks after a quiescent period is another important component (e.g., Lazzati et al. 2001; Connaughton 2002; Burrows et al. 2005).

Some authors argued that there are no obvious correlations between the precursors and the main peaks (Koshut et al. 1995; Lazzati 2005; Burlon et al. 2008, 2009; Charisi et al. 2015). Some others extracted and compared the temporal and spectral characteristics of EEs with main peaks (Norris et al. 2010, 2011; Bostanci et al. 2013; Kaneko et al. 2015; Kagawa et al. 2015; Lien et al. 2016; Anand et al. 2018). For example, Zhong et al. (2019) extracted 18 sGRB candidates with precursor observed by Fermi/GBM and Swift/BAT. They found that the average flux of precursor components tends to increase as those of the main peaks. They compared the hard ratio and the cutoff energy $E_{c}$ between these two emission episodes, suggesting that the main peaks are slightly harder than the precursors. Recently, Lan et al. (2020) identified 26 Fermi/GBM sGRBs with early EE similar to GRB 060614. Their results suggested that the sGRBs with EE probably have a similar physical origin. Particularly, they compared the properties of GRB 170817A as the first gravitational-wave associated sGRB with EE (Abbott et al. 2017; Goldstein et al. 2017), with other sGRBs with EE and found that there are no significant statistical differences between them.

The prompt GRB emissions are often produced by either the quasi-thermal mechanism from photosphere of a fireball or the synchrotron radiation mechanism from electrons of the Poynting-flux-dominated jet, respectively (Katz 1994; Meszaros et al. 1994; Thompson 1994; Rees \& Meszaros 1994; Daigne \& Mochkovitch 1998; Zhang \& Yan 2011; Deng \& Zhang 2014; Deng et al. 2015; Beniamini \& Giannios 2017; Lazarian et al. 2019; Meng et al. 2018, 2019; Li 2019b; Ryde et al. 2019). It is generally accepted that the low-energy photon index, is an indicator for the emission mechanism, using to distinguish the synchrotron and photosphere emissions (Li 2019a). Around 50\% of Swift GRBs are better explained by the black body (BB) spectrum for most X-ray flashes (XRFs) plus X-ray-rich GRBs (XRBs) or the synchrotron radiation mechanism for most classical GRBs (C-GRBs), correspondingly (Oganesyan et al. 2019; Zhang et al. 2020b). Lan et al. (2018) systematically studied the spectral and temporal properties of two emission episodes separated by quiescent gaps for $101 \mathrm{Fermi} / G B M$ IGRBs. They found similar distribution of peak energy between two emission episodes and suggested that these two share the same physical origin. However, it was found that the thermal component appears in the first emission episode and a transition from the thermal to the non-thermal component may 
exist in multipulse Fermi GRBs in the prompt gamma-ray emission phase (Zhang et al. 2018b; Li 2019a.b).

Zhang et al. (2018a) studied the time-resolved spectra in each episode of GRB 160625B detected by Fermi, from precursor to main peaks and to extended emission. They announced a transition from thermal to non-thermal mechanism within a GRB. The indisputable fact is that these investigations were mainly given to long bursts, not including short ones due to absence of sGRBs with multiple components. Recently, we defined two kinds of double-peaked BATSE sGRBs as M-loose and Mtight types according to their overlapping ratios between two adjacent main peaks (see Fig. 1 in Li et al. 2020). Then, we examined the temporal properties of the main peaks and the other two components of Swift/BAT sGRBs (Li et al. 2021). We adopted a united criterion to search for precursors and EEs as their signals prior and posterior to the main peaks at least $\mathrm{S} / \mathrm{N}>3$ above background (see section 2.2 in Li et al. 2021). Unfortunately, no such triplets have been reported in single sGRB to date. Considering the above controversial results, we generalize the spectral analysis and evolution of the three components by using sGRB samples with any two components instead. In this way, one can simultaneously investigate the time-integrated spectral properties of the three components of sGRBs with single or double main peaks. In addition, we will examine how the spectra evolve from precursor, main peak, to extended emission. Sample selection and spectra analysis are presented in Section 2. Section 3 displays our temporal and spectral results of these sGRBs. We end with the conclusions and discussions in Section 4.

\section{Data analysis method}

From 2004 December to 2019 July, Swift/BAT had detected 124 sGRBs, of which 26 sGRBs have been selected for this study, including 12 single-peaked sGRBs (SPs), 5 double-peaked sGRBs (DPs), 7 sGRBs with precursor (Pre+sGRBs), and 2 sGRBs with $\mathrm{EE}$ (sGRBs+EE). For the DPs, we still divide them into the Mtight types (Mt-DPs) and the M-loose ones (Ml-DPs) as done in our recent works ( $\mathrm{Li}$ et al. 2020, 2021). The criteria that we identify a significant precursor or EE pulse can be referred to our recent paper ( $\mathrm{Li}$ et al. 2021). The standard BAT software (HEADAS 6.26.1) and the latest calibration database (CALDB: 2017-10-16) are used. Refer to BAT analysis threads 1 for the handing process.

\footnotetext{
1 https://swift.gsfc.nasa.gov/analysis/threads/ bat_threads.html/ or https://www.swift.ac.uk/analysis/ bat/
}
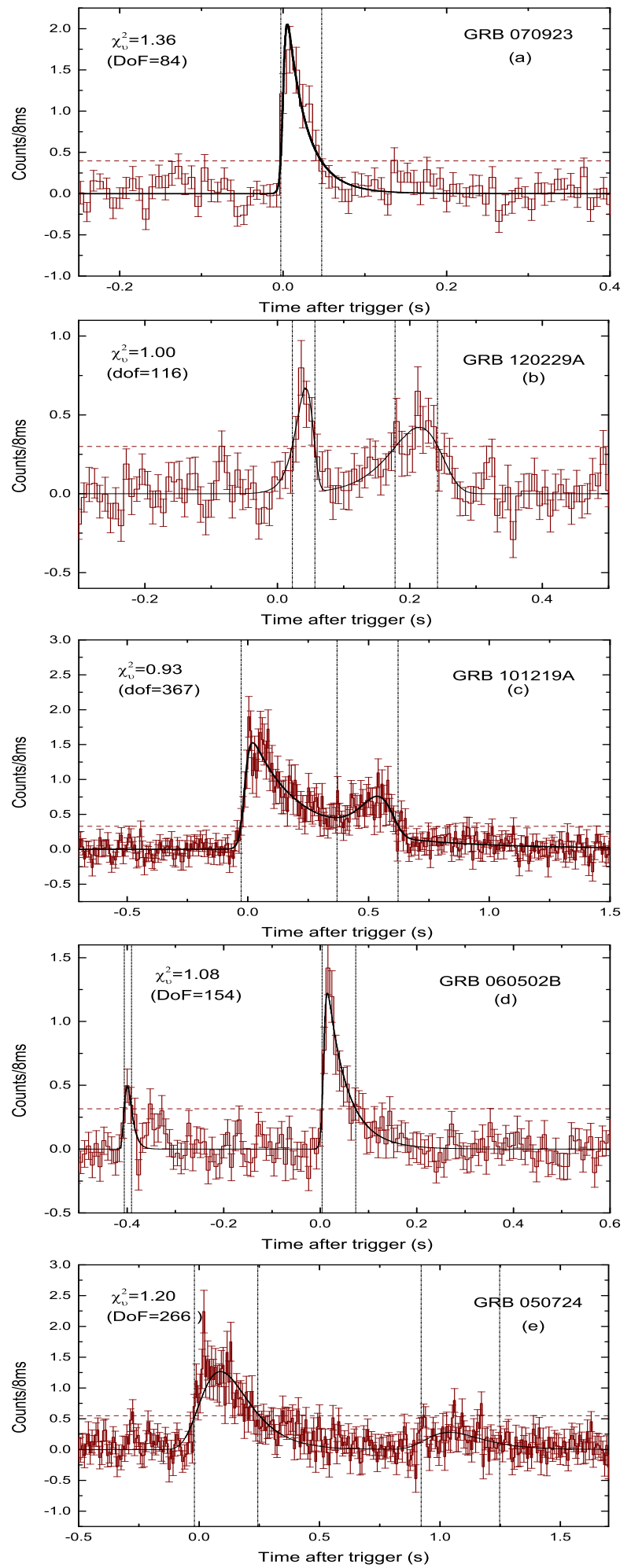

Fig. 1. The light curves (15-350KeV) of five typical sGRBs. The horizontal dashed lines mark a $3 \sigma$ confidence level. The vertical dashed lines show the starting and the ending times of the emission target pulses. (a) single pulse; (b) M-loose; (c) M-tight; (d) Main peak with precursor; (e) Main peak with EE.

The mask-weighted light curve data of the sGRBs with a 8-ms resolution are taken from the Swift website (Lien et al. 2016) 2. Note that all light curves of the selected 26 Swift/BAT sGRBs can be well fitted by the empirical Kocevski-Ryde-Liang

2 https://swift.gsfc.nasa.gov/results/batgrbcat/ 
(KRL) function that has been popularly used in literatures (e.g., Kocevski et al. 2003; Zhang \& Oin 2005; Li et al. 2020, 2021). Fig. 1 shows five representative cases that can be fitted successfully according to the reduced Chi-square standard together with a residual assessment (Li et al. 2020, 2021). The KRL function with five free parameters is

$f(t)=f_{m}\left(\frac{t+t_{0}}{t_{m}+t_{0}}\right)^{r}\left[\frac{d}{d+r}+\frac{r}{d+r}\left(\frac{t+t_{0}}{t_{m}+t_{0}}\right)^{(r+1)}\right]^{-\left(\frac{r+d}{r+1}\right)}$,

where $r$ and $d$ respectively determine the rise and the decay shapes of an individual pulse, $f_{m}$ represents the peak flux, $t_{m}$ is the peak time, $t_{0}$ is the offset of the pulse from the trigger time. The fitting processes have been conducted in energy channel 15$350 \mathrm{keV}$. We define the duration of an emission segment with $t_{d u r}=t_{e}-t_{s}$, in which $t_{s}$ and $t_{e}$ are the starting and the ending times of a given pulse at the level of $\mathrm{S} / \mathrm{N}=3$. Based on the above fitting with Eq. 1, one can easily obtain all temporal features of different kinds of sGRBs. Finally, our sample includes 42 GRB pulses, of which 33, 7 and 2 pulses have been taken from main peaks, precursors and EEs, respectively. The fitting parameters are listed in Appendix A Table1.

Subsequently, we utilize software XSPEC to perform the model fitting of the spectrum of each episode as shown in Fig. 1. A power-law (PL) or cutoff power-law (CPL) spectral form has been applied to fit the GRB spectra because the Swift/BAT has a narrow energy band (see also Zhang et al. 2020b). The PL model is written as

$N_{E, P L}(E)=N_{0, P L} E^{-\alpha_{p l}}$,

where $N_{0, P L}$ is the photon flux (photons $\mathrm{cm}^{-2} \mathrm{keV}^{-1} \mathrm{~s}^{-1}$ ), $E$ is the photon energy, $\alpha_{p l}$ is the low photon spectral index. The CPL model can be written as

$N_{E, C P L}(E)=N_{0, C P L} E^{-\alpha_{c p l}} \exp \left(-E / E_{p}\right)$,

in which $N_{0, C P L}$ is the photon flux (photons $\mathrm{cm}^{-2} \mathrm{keV}^{-1} \mathrm{~s}^{-1}$ ), $E$ is the photon energy, $\alpha_{c p l}$ is the low photon spectral index, $E_{p}$ is the peak energy in keV. Besides, a Planck black-body (BB) function used to identify the thermal component can be expressed by

$N_{E, B B}(E)=N_{0, B B} \frac{8.0525 E^{2} d E}{(k T)^{4}[\exp (E / k T)-1]}$,

where $k T$ is the thermal energy of electrons and $E$ is the photon energy, both energies are in units of keV. Note that the model is built in Xspec.

The reduced $\chi_{v}^{2}$ is given to estimate the goodness of spectrum fitting. We choose CPL if $\Delta \chi^{2}=\chi_{P L}^{2}-\chi_{C P L}^{2}>6$ and PL if $\chi_{P I}^{2}-\chi_{C P I}^{2} \leq 6$ as the best-fit model (Sakamoto et al. 2009; Lien et al. 2016; Katsukura et al. 2020). This criterion is used in the BAT team for reporting the spectral parameters based on a CPL fit. Totally, Appendix A Table 2 lists the results of the temporal and spectral properties of 26 typical Swift/BAT sGRBs. Column (1) lists the GRB name; Column (2) lists the duration $T_{90}$; Column (3) lists the cosmological redshift; Column (4) lists the duration $t_{d u r}$ of each pulse; Columns (5) - (10) respectively represent the observed peak energy $E_{p}$, the spectral index $\alpha$, the average flux in unit of erg $\mathrm{cm}^{-2} \mathrm{~s}^{-1}$, the observed energy fluence in unit of erg cm $\mathrm{cm}^{-2}$, and the goodness of spectrum fitting for the PL model; While columns (11) - (16) display the corresponding parameters for the CPL model. Finally, Columns (17) and (18) show the $\Delta \chi^{2}=\chi_{P L}^{2}-\chi_{C P L}^{2}$ and the best model. Note that we use the time of the valleys as the boundary of overlapping pulses in Mt-DPs. Because of relatively weaker EE signal, the duration of
sGRB 050724 is defined in terms of the time domain when the fitted intensity is equal to $\mathrm{e}^{-1}$ of its maximum value. And note that our sample also includes the first gravitational-wave associated GRB 170817A detected by Fermi/GBM (Goldstein et al. 2017; Savchenko et al. 2017). Zhang et al. (2018a) reported the detailed temporal and spectral properties for the main peaks and the EE components. Using their results, we compare the properties of GRB 170817A with those of the other typical sGRBs in our samples thus it is beneficial to identifying the candidates similar to GRB 170817A.

\section{Results}

In this section, we present the main results of temporal and spectral parameters such as pulse durations $\left(t_{d u r}\right)$, peak energy $\left(E_{p}\right)$, average flux $\left(F_{p}\right)$, and energy fluence $\left(S_{\gamma}\right) 3$ together with their correlations and evolutions.

\subsection{Spectral characteristics of diverse prompt emission segments}

Most Swift/BAT GRB spectra can be fitted by a simple PL due to the narrow energy band (Zhang et al. 2007ab; Sakamoto et al. 2011). Previous studies illustrated that the relation $\alpha_{p l}-E_{p}$ can be employed as an indicator to estimate the $E_{p}$ of a burst without good spectral breaks (Crider et al. 1997; Kaneko et al. 2006; Zhang et al. 2007b; Sakamoto et al. 2009; Virgili et al. 2012). This relation was first derived by Zhang et al. (2007b) and then confirmed by Virgili et al. (2012) as

$\log E_{p}=(2.76 \pm 0.07)-(3.61 \pm 0.26) \log \alpha_{p l}$,

with a varied low energy spectral index of $1.2 \leq \alpha_{p l} \leq 2.3$. Similar to the conclusion of Sakamoto et al. (2009), the bursts with $\alpha_{p l}<1.2$ should have a higher $E_{p}$ far beyond the Swift/BAT band while the bursts with $\alpha_{p l}>2.3$ are likely X-ray flashes with $E_{p}$ near or below the low-energy end of Swift/BAT (Zhang et al. $2007 \mathrm{a}$ b).

In spite of the Eq. 5 is heavily dominated by lGRBs, the sGRBs are generally consistent with the relationship (see Fig. 2 in Zhang et al. 2007b). Additionally, using a completely new GRB sample, including 31 short and 252 long GRBs with wellmeasured peak energy and redshift, Zhang et al. (2018a) found that short and long GRBs hold the coincident $E_{p, i}-E_{i s o}$ correlations, indicating that both kinds of GRBs may share the same radiation mechanism, which is consistent with the conclusion of Minaev \& Pozanenko (2020). Consequently, we assume that Eq. 5 is also available for sGRBs, together with their isolated emission components. Note that about $71 \%(30 / 42)$ of the segmental spectra can be well fitted by the PL. There are 16 segments with $1.2 \leq \alpha_{p l} \leq 2.3$, whose $E_{p}$ can be ideally fitted by the Eq. 5 .

\subsubsection{GRB spectra in pulse durations}

We estimate the $E_{p}$ within each $t_{d u r}$ of 30 isolated segments by way of either the Eq. 5 for the PL spectra or the CPL fitting directly. Fig. 2a shows that the pulse durations are lognormally distributed with a mean value of $0.12 \pm 0.02 \mathrm{~s}$. It is found that from Fig. 2b $E_{p}$ and $t_{d u r}$ are uncorrelated with each other due to a much lower correlation coefficient of 0.14 . This is largely different from the anti-correlation of $E_{p}$ with $T_{90}$ between sGRBs

3 The fluence is calculated in the energy range of $15-350 \mathrm{keV}$. 


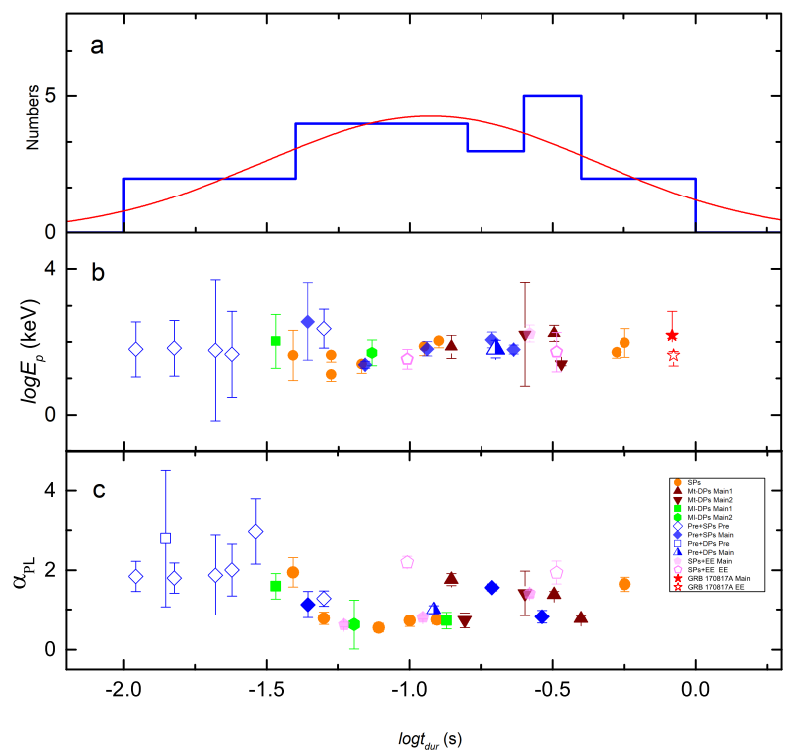

Fig. 2. Panel a: distribution of $\mathrm{t}_{d u r}$ for 30 segments with $E_{p}$ measurement; Panel b: $E_{p}$ is plotted against $t_{d u r}$ for 30 segments with $E_{p}$ measurement; Panel c: $\alpha_{P L}$ is plotted against $t_{d u r}$ for 30 segments. The main peaks are marked with filled shapes. The precursors or the EEs are symbolized with empty shapes.

and IGRBs (e.g., Zhang \& Choi 2008). Moreover, we also analyze the relation between low energy spectral index $\alpha$ and pulse width in Fig. 2c, where no any correlation is found among them. No obviously spectral evolution across different prompt $\gamma$-ray components are manifested in Fig. $2 b$ and $2 c$ for the sGRBs. In general, the spectrum of late-time EEs is relatively softer than that of main peaks. However, almost all the EE segments identified in our sample occur within 2 seconds since the trigger time. It can be understood that the prompt sGRB spectra do not evolve in a very short period.

\subsubsection{Flux versus fluence}

Fig. 3 shows a tight correlation between $F_{p}$ and $S_{\gamma}$. The correlation for our selected sGRB sample is

$\log F_{p}=(0.62 \pm 0.07) \log S_{\gamma}+(0.27 \pm 0.07)$,

with a Pearson correlation coefficient $\rho=0.83$ and a chance probability $P=5.6 \times 10^{-12}$. It is necessary to announce that this correlation is valid only when all event pulses (including GRB 170817A) in our sample are considered. Noticeably, the values of $F_{p}$ and $S_{\gamma}$ of the precursors are relatively lower than those of other components of sGRBs. It is worth nothing that GRB $170817 \mathrm{~A}$ as an off-axis sGRB is marginally coincident with Eq. 6 and will affect this correlation slightly when it is ignored.

\subsubsection{Peak energy versus fluence}

Recently, we studied the observed $E_{p}-S_{\gamma}$ relations of 283 Swift GRBs comprising 252 lGRBs and 31 sGRBs with known redshift and measured $E_{p}$ and found that sGRBs and lGRBs are differently distributed in the plane of $E_{p}$ versus $\mathrm{S}_{\gamma}$ (Zhang et al. 2018). More recently, Zhang et al. (2020b) proposed a useful correlation of $E_{p} \sim S_{\gamma}^{0.28}$ that can be applied as an $E_{p}$ indicator for those bursts with unknown $E_{p}$ (Zhang et al. 2020b). Motivated by these results, we now focus on the analysis of the same correlation for the diverse emission segments in sGRBs whose light curves can be well fitted as shown in Fig. 1. It is surprisingly shown in Fig. 4 that there is no obvious correlation between $E_{p}$ and $S_{\gamma}$ with a Pearson coefficient of 0.16 and a chance probability of 0.39 , which is primarily resulted from the independence of peak energy on pulse duration as exhibited in Fig. 2. The solid and dashed lines show the empirical relation of $E_{p}$ and $S_{\gamma}$ of the sGRBs and lGRBs with well measured spectrum proposed by Zhang et al. (2020b). Note that GRB 170817A resides among the sGRB group, which is much similar to the finding for 31 sGRBs with known redshift by Zhang et al. (2018) and the short or type E-II GRBs with EE in Zhang et al. (2020a).

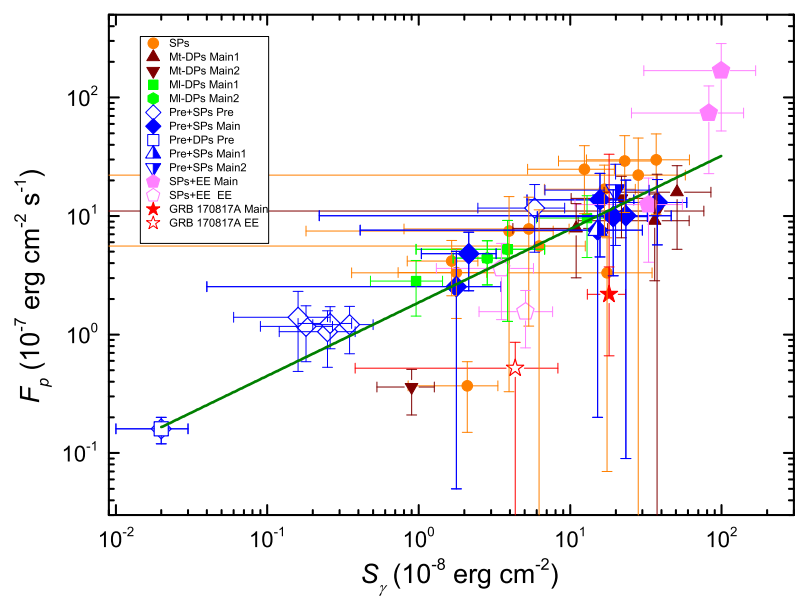

Fig. 3. $F_{p}$ is plotted against $S_{\gamma}$ for 44 pulses including GRB 170817A in our sample. The olive line denotes the best logarithmic fit. All symbols are same as in Fig. 2.

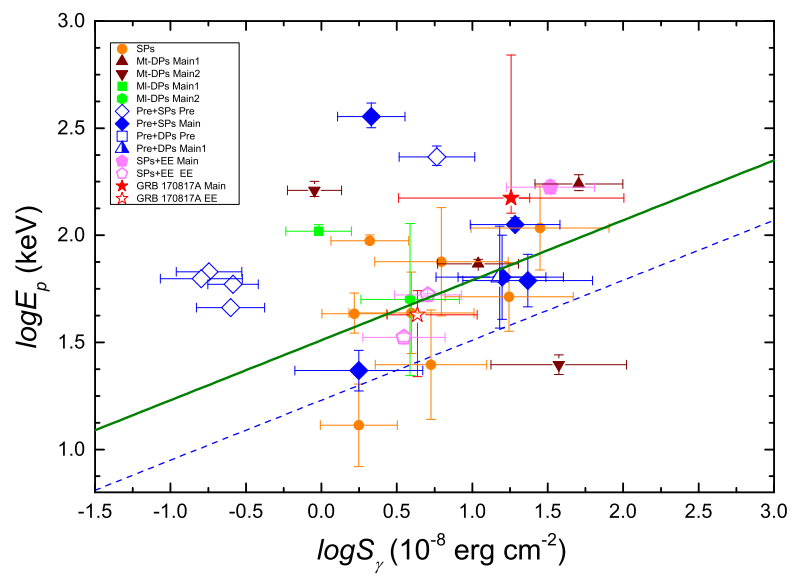

Fig. 4. $E_{p}$ is plotted against $S_{\gamma}$ for 30 segments including GRB 170817A. The solid olive and dashed royal lines are the best logarithmic fit to the correlation between $S_{\gamma}$ and $E_{p}$ for the sGRBs and lGRBs in Zhang et al. (2020b). All symbols are same as in Fig. 2.

\subsection{Spectral evolution}

\subsubsection{Radiative intensity of diverse segments}

In order to test whether the spectra of sGRBs evolve from the early components to the later one in the phase of prompt $\gamma$-ray emissions, we compare the peak flux $F_{p}$ of the early pulse $\left(F_{p, e}\right)$ with the later pulse $\left(F_{p, l}\right)$, as well as other key spectral parameters $\left(E_{p}\right.$ and $\left.S_{\gamma}\right)$. 


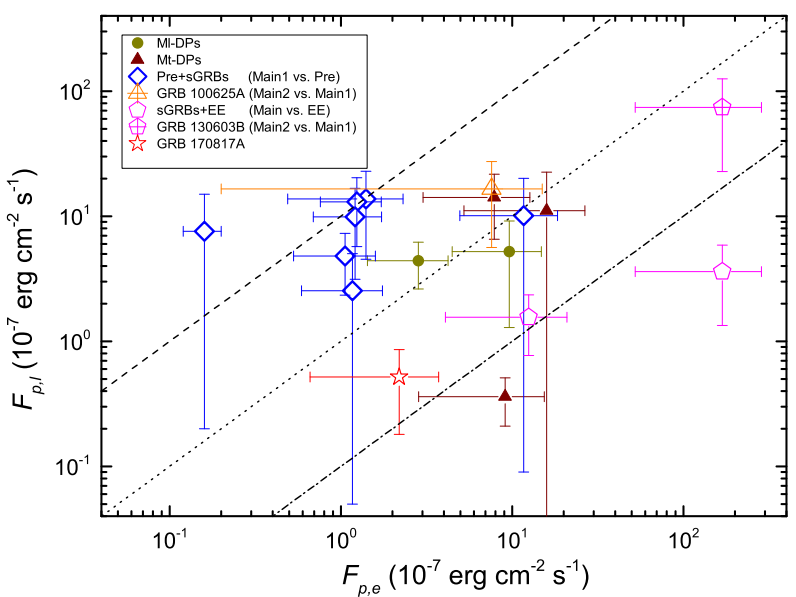

Fig. 5. $F_{p}$ comparisons between the early and the later pulses in the one-component (two-type DPs, filled symbols) and the two-component (empty symbols) sGRBs. Three peak flux ratios of $F_{p, l}$ to $F_{p, e}$ are signified by the dashed, dotted and dash-dotted lines for 10,1 and $1 / 10$, respectively.

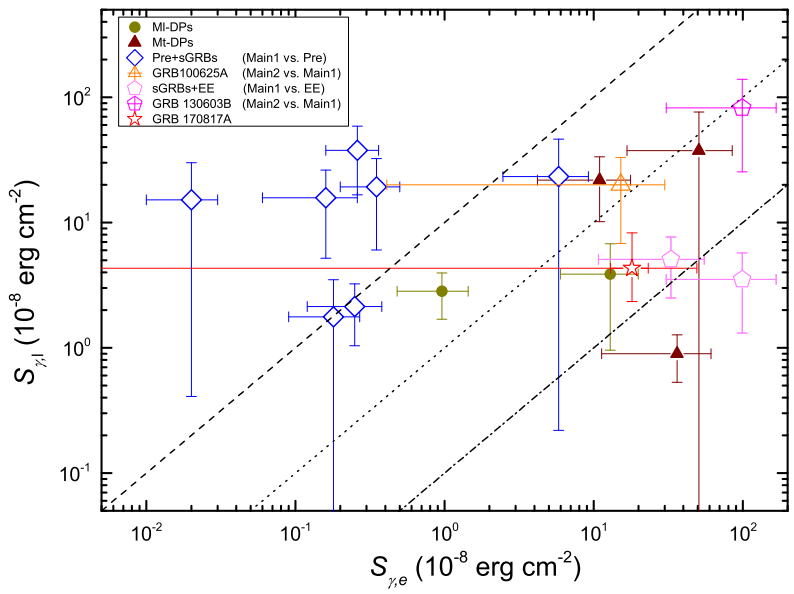

Fig. 6. Comparison between $S_{\gamma}$ of the early and the later pulses in the one-component (two-type DPs, filled symbols) and the two-component (empty symbols) sGRBs. Three peak flux ratios of $S_{\gamma, l}$ to $S_{\gamma, e}$ are signified by the dashed, dotted and dash-dotted lines for 10,1 and $1 / 10$, respectively.

We compare the peak flux densities of main peaks (filled symbols) with both precursors and EEs (empty symbols) in Fig. 5] where it can seen that the main peaks are on average brighter than the other two components about one order of magnitude (see also Zhang et al. 2020a). In addition, two types of DPs lying near to the dotted line, shows that the brightness of two main peaks are comparable with each other, which is consistent with the conclusion drawn by Lan et al. (2018). Based on an analysis of Pre+Mt-DP 100625A and Mt-DP+EE 130603B, we find that the peak fluxes of two main peaks are similar to those isolated Mt-DPs. Furthermore, we find the similar results for the observed fluence $S_{\gamma}$ in Fig. 6 .

\subsubsection{Features of spectral evolution}

Zhang et al. (2007a) reported that the burst with $\alpha_{p l}>2.3$ is likely a softer $\gamma$ event called XRF with peak energy near or below the low-energy end of BAT. In this case, Eq.5 cannot be used to estimate the $E_{p}$. For two precursors with $\alpha_{p l}>2.3$ of GRBs
100702A and 100625A, we try to invoke the Planck black-body model (Eq. 4) to fit the spectra and obtain the thermal energy of electrons $K T=6.02 \pm 1.57 \mathrm{KeV}$ and $K T=9.04 \pm 1.98 \mathrm{KeV}$, indicating that the thermal contributions to GRB spectra are negligible. On the other hand, it is found that there are 12 main peaks whose best models are PL with $\alpha_{p l}<1.2$, including SPs, Pre+SPs, Pre+DPs and sGRB+EE. In this situation, Eq. 5 cannot be used to also estimate the $E_{p}$ effectively, since the $E_{p}$ will be quite outside of the BAT band. Moreover, we find that the EEs of GRBs 050724 and 130603B are slightly softer than their main peaks, which is consistent with some previous conclusions (e.g., Norris \& Bonnell 2006; Norris et al. 2010; Kagawa et al. 2015). However, we emphasize that the spectra of sGRBs in our sample do not evolve during prompt $\gamma$ ray emission epoch, which challenges the known theoretical models for the precursors and the EEs (e.g. Murakami et al. 1991; Lyutikov \& Usov 2000; Metzger et al. 2011). On the other hand, the non-evolutionary phenomena can be supported by the zero lags of light curves between different energy channels for sGRBs (e.g. Norris et al. 2006; Zhang et al. 2006). By looking back to Fig. 2, we can conclude that the parameters $E_{p}$ and $\alpha$ are two representative qualities describing the spectral evolution consistently (see also Ghirlanda et al. 2004).

\section{Conclusions and Discussions}

We summarize the major results as follows.

(1) We find that the peak energies of diverse $\gamma$-ray radiation segments in sGRBs with single or double main peaks are uncorrelated with the corresponding $t_{d u r}$.

(2) We find a tight correlation between $F_{p}$ and $S_{\gamma}$ for different segments to be $\log F_{p}=(0.62 \pm 0.07) \log S_{\gamma}+(0.27 \pm 0.07)$.

(3) In the plane of $E_{p}$ versus $S_{\gamma}$, these diverse $\gamma$-ray radiation segments in sGRBs distribute near to the line of $E_{p} \sim S_{\gamma}^{0.28}$ found by Zhang et al. (2020b) for those sGRBs with a well-measured spectrum. However, there is no obvious correlation found between $E_{p}$ and $S_{\gamma}$ for these segments entirely.

(4) The main peaks are on average brighter than the precursors or EEs about one order of magnitude. Regarding the EEs, our result is consistent with Zhang et al. (2020a).

(5) In terms of the analyses of peak energies and low spectral index of diverse $\gamma$-ray radiation segments, it is found that the sGRB spectra of precursors, main peaks and EEs exhibit no obvious evolutionary sequence.

Unfortunately, since the absences of the EEs or precursors might be related to sensitivity or energy coverage of the current GRB detectors, no such sGRBs with three distinct components have been observed. For example, though the Fermi/GBM with a broader energy band had identified over 2000 GRBs (von Kienlin et al. 2020), only 4 of 244 precursors are identified in sGRBs (Coppin et al. 2020). Fortunately, more and more GRB monitors have been launched or planned to launch to meet the increasing requirements of the X-ray/gamma-ray counterpart observation. More than $200 \mathrm{GRBs}$ were detected by Chinese first $\mathrm{X}$-ray astronomical satellite Hard X-ray Modulation Telescope (HXMT), thanks to its wider energy coverage from $1 \mathrm{keV}$ to $3 \mathrm{MeV}$, large filed of view, and good sensitivity (Zhang et al. 2020c; Liu et al. 2020). The Gravitational wave high-energy Electromagnetic Counterpart All-sky Monitor (GECAM) which has an all-sky field of view, a high sensitivity and a wide energy interval (6 keV - $5 \mathrm{MeV})$ has been launched in 2020 (Liao et al. 2020; Song et al. 2020; Chen et al. 2020). Meanwhile, Space multi-band astronomical Variable Objects Monitor (SVOM) whose energy range is from $15 \mathrm{keV}$ to $5 \mathrm{MeV}$ aims at detecting very distant and faint/soft nearby GRBs. SVOM with 
rapid slew capability will provide GRB positions and spectral parameters on very short time scale in the near decade through a collection of instruments in various gamma and $\mathrm{X}$ energy bands as well as in visible wave lengths through a narrow field of view telescope (Wei et al. 2016). Hopefully, our results can shed new light on the studies of physical processes of sGRBs. Meanwhile, further search for three-components sGRBs simultaneously from the Fermi, HXMT, GECAM and SVOM catalogs, can draw more robust conclusions in the future.

Acknowledgements. We thank the anonymous referee for very helpful suggestion and constructive comments. This work was supported by the National Natural Science Foundation of China (No. U2031118), the Youth Innovations and Talents Project of Shandong Provincial Colleges and Universities (Grant No. 201909118) and the Natural Science Foundations (ZR2018MA030, XKJJC201901).

\section{References}

Abbott, B. P., Abbott, R., Abbott, T. D., et al. 2017, ApJL, 848, L13 Anand, N., Shahid, M., \& Resmi, L., 2018, MNRAS, 481, 4332

Beniamini, P., \& Giannios, D., 2017, MNRAS, 468, 3202

Bernardini, M.G., Campana, S., Ghisellini, G., et al. 2013, ApJ, 775, 67

Burlon, D., Ghirlanda, G., Ghisellini, G., et al. 2008, ApJ, 685, L19

Burlon, D., Ghirlanda, G., Ghisellini, G., et al. 2009, A\&A, 505, 569

Burrows, D.N., Romano,P., Falcone, A., et al. 2005, Science, 309, 1833

Bostanc1, Z.F., Kaneko, Y., \& Göğüs, E., 2013, MNRAS, 428, 1623

Charisi, M., Marka, S., \& Bartos, I., 2015, MNRAS, 448, 2624

Chen, W., Song, L.M., Zheng, S.J. et al. 2020, Sci. China-Phys. Mech. Astron. 50,129512

Colgate, S.A., 1974, ApJ, 187, 333

Connaughton, V. 2002, ApJ, 567, 1028

Coppin, P., de Vries, K. D. \& van Eijndhoven, N. 2020, Physical Review D, 102, 103014

Crider, A., Liang, E.P., Smith, I.A., et al. 1997, ApJL, 479, L39

Daigne, F., \& Mochkovitch, R., 1998, MNRAS, 296, 275

Deng, W., \& Zhang, B., 2014, ApJ, 785, 112

Deng, W., Li, H., Zhang, B., et al. 2015, ApJ, 805, 163

Dereli-Bégué, H., Pe'er, A., \& Ryde, F., 2020, ApJ, 897, 145

Gehrels, N., Chincarini, G., Giommi, P., et al. 2004, ApJ, 611, 1005

Ghirlanda, G., Ghisellini, G. \& Celotti, A., 2004, A\&A, 422, L55

Goldstein, A., Veres, P., Burns, E., et al. 2017, ApJL, 848, L14

Hu, Y.-D., Liang, E.-W., Xi, S.-Q., et al. 2014, ApJ, 789, 145

Kagawa, Y., Yonetoku, D., Sawano, T., et al. 2015, ApJ, 811, 4

Kaneko, Y., Preece, R.D., Briggs, M.S., et al. 2006, ApJS, 166, 298

Kaneko, Y., Bostanc1, Z.F., Göğüş, E., et al. 2015, MNRAS, 452, 824

Katsukura, D., Sakamoto, T., Tashiro, M.S., et al. 2020, ApJ, 889, 110

Katz, J.I., 1994, ApJ, 422, 248

Kocevski, D., Ryde, F., \& Liang, E., 2003, ApJ, 596, 389

Koshut, T.M., Konveliotou, C., Paciesas, S.W., et al. 1995, ApJ, 452, 145

Kouveliotou, C., Meegan, C.A., Fishman, G.J. et al. 1993, ApJL, 413, L101

Lan, L., Lü, H.-J., Zhong, S.-Q., et al. 2018, ApJ, 862, 155

Lan, L., Lu, R.-J., Lü, H.-J. et al. 2020, MNRAS, 492, 3622

Lazarian, A., Zhang, B., \& Xu, S., 2019, ApJ, 882, 184

Lazzati, D., Ramirez-Ruiz, E., \& Ghisellini, G. 2001, A\&A , 379, L39

Lazzati, D., 2005, MNRAS, 357, 722

Li, L., 2019a, ApJS, 242, 16

Li, L., 2019b, ApJS, 245, 7

Liao, J.Y., Luo, Q., Zhu, Y. et al. 2020, Sci. China-Phys. Mech. Astron. 50, 129510

Lien, A., Sakamoto, T., Barthelmy, S.D., et al. 2016, ApJ, 829, 7

Liu, C.Z., Zhang, Y.F., Li, X. F., et al. 2020, Sci. China-Phys. Mech. Astron. 63, 249503

Li, X.J., Zhang, Z.B., Zhang, C.T., et al. 2020, ApJ, 892, 113

Li, Y., Zhang, B., \& Yuan, Q., 2020, ApJ, 897, 154

Li, X.J., Zhang, Z.B., Zhang, X.L., et al. 2021, ApJS, 252, 16

Lyutikov, M., \& Usov, V.V., 2000, ApJ, 543, L129

Metzger, A.E., Parker, R.H., Gilman, D., et al. 1974, ApJL, 194, L19

Metzger, B. D., Giannios, D., Thompson, T. A., et al. 2011, MNRAS, 413, 2031.

Meng, Y.-Z., Geng, J.-J., Zhang, B.-B., et al. 2018, ApJ, 860, 72

Meng, Y.-Z., Liu, L.-D., Wei, J.-J., et al. 2019, ApJ, 882, 26

Meszaros, P., Rees, M.J., \& Papathanassiou, H., 1994, ApJ, 432, 181

Minaev, P. Y. \& Pozanenko, A. S. 2020, MNRAS, 492, 1919.

Murakami, T., Inoue, H., Nishimura, J., et al. 1991, Nature, 350, 592

Norris, J.P., \& Bonnell, J.T., 2006, ApJ, 643, 266

Norris, J.P., Gehrels, N., \& Scargle, J.D., 2010, ApJ, 717, 411
Norris, J.P., Gehrels, N., \& Scargle, J.D., 2011, ApJ, 735, 23

Oganesyan, G., Nava, L., Ghirlanda, G., et al. 2019, A\&A, 628, A59

Preece, R.D., Briggs, M.S., Mallozzi, R.S., et al. 1998, ApJL, 506, L23

Rees, M.J., \& Meszaros, P., 1994, ApJL, 430, L93

Ryde, F., Yu, H.-F., Dereli-Bégué, H., et al. 2019, MNRAS, 484, 1912

Sakamoto, T., Sato, G., Barbier, L., et al. 2009, ApJ, 693, 922

Sakamoto, T., Barthelmy, S.D., Baumgartner, W.H., et al. 2011, ApJS, 195, 2

Savchenko, V., Ferrigno, C., Kuulkers, E., et al. 2017, ApJL, 848, L15

Song, X.Y., Xiong, S.L., Luo, Q. et al. 2020, Sci. China-Phys. Mech. Astron. 50, 129511

Thompson, C., 1994, MNRAS, 270, 480

Troja, E., Rosswog, S., \& Gehrels, N., 2010, ApJ, 723, 1711

Virgili, F.J., Qin, Y., Zhang, B., et al. 2012, MNRAS, 424, 2821

von Kienlin, A., Meegan, C.A., Paciesas, W.S., et al. 2020, ApJ, 893, 46

Wei, J., Cordier, B., Antier, S. et al. arXiv:1610.06892

Zhang, B., Liang, E.W., Page, K.L., et al. 2007a, ApJ, 655, 989

Zhang, B., Zhang, B.-B., Liang, E.-W., et al. 2007b, ApJL, 655, L25

Zhang, B., \& Yan, H.R., 2011, ApJ, 726, 90

Zhang, B.-B., Zhang, B., Sun, H.,et al. 2018a, Nature Communications, 9, 447

Zhang, B.-B., Zhang, B., Castro-Tirado, A.J., et al. 2018b, NatAs, 2, 69

Zhang, S.-N., Li, T.P., Lu, F.J., et al. 2020c, Sci. China-Phys. Mech. Astron. 63, 249502

Zhang, X.L., Zhang, C.T., Li, X.J., et al. 2020a, RAA, 20, 201

Zhang, Z.B., \& Qin, Y.P., 2005, MNRAS, 363, 1290

Zhang, Z.B., Xie, G.Z., Deng, J. G., et al. 2006, MNRAS, 373, 729

Zhang, Z.B., \& Choi, C.S. 2008, A\&A, 484, 293

Zhang, Z.B., Zhang, C.T., Zhao, Y.X. et al. 2018, PASP, 130, 054202

Zhang, Z.B., Jiang, M., Zhang, Y., et al. 2020b, ApJ, 902, 40

Zhong, S.-Q., Dai, Z.-G., Cheng, J.-G., et al. 2019, ApJ, 884, 25

\section{Appendix A:Tables}

Article number, page 6 of 8 
Table 1. Fitting parameters of all individual pulses in Swift sGRBs.

\begin{tabular}{|c|c|c|c|c|c|c|c|}
\hline \multirow{2}{*}{$\begin{array}{l}\overline{G R B} \\
\overline{\text { SPs }}\end{array}$} & \multirow[t]{2}{*}{$\overline{f_{m}}$} & \multirow[t]{2}{*}{$\overline{\overline{t_{m}}}$} & \multirow[t]{2}{*}{$\mathrm{r}$} & \multirow[t]{2}{*}{$\overline{\mathrm{d}}$} & \multirow[t]{2}{*}{$\overline{t_{0}}$} & \multicolumn{2}{|c|}{$\overline{D O O F \quad \chi^{2} / D O F}$} \\
\hline & & & & & & & \\
\hline 070923 & $2.053 \pm 0.483$ & $.005 \pm 0.004$ & $155.833 \pm 177.285$ & $13.529 \pm 2.156$ & $0.300 \pm 0.000$ & 84 & 1.36 \\
\hline $090621 \mathrm{~B}$ & $1.106 \pm 0.139$ & $0.053 \pm 0.006$ & $70.672 \pm 1055.989$ & $34.488 \pm 459.488$ & $1.127 \pm 16.704$ & 97 & 1.13 \\
\hline $100206 \mathrm{~A}$ & $1.785 \pm 0.144$ & $0.039 \pm 0.006$ & $22.181 \pm 118.060$ & $10.749 \pm 39.672$ & $0.400 \pm 2.004$ & 90 & 0.90 \\
\hline $110420 \mathrm{~B}$ & $2.601 \pm 3.424$ & $0.002 \pm 0.020$ & $(-2.627 \pm 91.410) E 14$ & $(-3.722 \pm 12.307) E 12$ & $(-1.209 \pm 4.090) E 11$ & 73 & 1.21 \\
\hline $120305 \mathrm{~A}$ & $5.831 \pm 0.294$ & $0.020 \pm 0.001$ & $63.766 \pm 7.074$ & $11.159 \pm 0.629$ & $0.300 \pm 0.000$ & 85 & 1.23 \\
\hline $131004 \mathrm{~A}$ & $0.661 \pm 0.035$ & $0.051 \pm 0.018$ & $3.537 \pm 0.515$ & $2.748 \pm 0.372$ & $0.500 \pm 0.000$ & 248 & 1.11 \\
\hline $140622 \mathrm{~A}$ & $0.717 \pm 0.126$ & $0.016 \pm 0.009$ & $14.893 \pm 79.699$ & $3.965 \pm 10.719$ & $0.151 \pm 0.678$ & 176 & 0.96 \\
\hline $150301 \mathrm{~A}$ & $3.085 \pm 0.237$ & $0.006 \pm 0.001$ & $175.122 \pm 46.756$ & $15.547 \pm 1.640$ & $0.290 \pm 0.000$ & 59 & 0.94 \\
\hline 150710 & $2.356 \pm 1.744$ & $0.016 \pm 0.016$ & $(3.732 \pm 18.496) E 14$ & $(6.923 \pm 6.081) E 12$ & $(2.607 \pm 2.116) E 11$ & 71 & 1.19 \\
\hline $160601 \mathrm{~A}$ & $0.875 \pm 0.159$ & $0.056 \pm 0.008$ & $4.582 \pm 3.901$ & $(-1.211 \pm 3.133) E 13$ & $0.181 \pm 0.165$ & 96 & 0.98 \\
\hline$\overline{180727 \mathrm{~A}}$ & $0.587 \pm 0.036$ & $0.505 \pm 0.018$ & $3.769 \pm 0.227$ & $(-1.497 \pm 1.246) E 13$ & $0.900 \pm 0.000$ & 434 & 1.07 \\
\hline $190326 \mathrm{~A}$ & $4.922 \pm 0.548$ & $0.006 \pm 0.002$ & $263.792 \pm 1148.656$ & $15.800 \pm 58.584$ & $0.409 \pm 1.669$ & 90 & 1.00 \\
\hline \multicolumn{8}{|l|}{$\overline{\overline{\text { Mt-DPs }}}$} \\
\hline \multirow[t]{2}{*}{$101219 \mathrm{~A}$} & $1.527 \pm 0.092$ & $0.020 \pm 0.007$ & $63.213 \pm 48.603$ & $3.882 \pm 1.583$ & $0.728 \pm 0.437$ & 367 & 0.93 \\
\hline & $0.551 \pm 0.145$ & $0.541 \pm 0.009$ & $19.311 \pm 2.346$ & $(-8.259 \pm 1.398) E 14$ & $0.761 \pm 0.158$ & - & - \\
\hline \multirow[t]{2}{*}{$\overline{120804 \mathrm{~A}}$} & $1.447 \pm 0.129$ & $0.106 \pm 0.010$ & $-5.132 \pm 0.528$ & $(-8.984 \pm 1.414) E 13$ & $0.999 \pm 0.132$ & 379 & 1.06 \\
\hline & $1.718 \pm 0.214$ & $0.402 \pm 0.007$ & $25.034 \pm 11.678$ & $(-2.131 \pm 2.000) E 14$ & $1.002 \pm 0.622$ & - & - \\
\hline \multirow[t]{2}{*}{$130912 \mathrm{~A}$} & $1.952 \pm 0.501$ & $0.033 \pm 0.003$ & $-22.117 \pm 30.011$ & $(-1.537 \pm 1.981 E 14$ & $0.487 \pm 0.686$ & 115 & 1.19 \\
\hline & $0.794 \pm 0.390$ & $0.271 \pm 0.008$ & $18.102 \pm 10.586$ & $(-1.448 \pm 1.966) E 14$ & $0.631 \pm 0.494$ & - & - \\
\hline \multicolumn{8}{|l|}{$\overline{\overline{\text { Ml-DPs }}}$} \\
\hline \multirow[t]{2}{*}{$120229 \mathrm{~A}$} & $650 \pm 0.150$ & $.043 \pm 0.006$ & 284.368 & $(-1.13$ & $0.986 \pm 3.571$ & 116 & 1.00 \\
\hline & $0.421 \pm 0.081$ & $0.215 \pm 0.006$ & $25.489 \pm 2.742$ & $(-1.851 \pm 0.206) E 15$ & $0.772 \pm 0.101$ & - & - \\
\hline \multirow[t]{2}{*}{$111117 \mathrm{~A}$} & $0.439 \pm 0.099$ & $0.107 \pm 0.014$ & $-8.705 \pm 4.986$ & $(-1.139 \pm 0.717) E 14$ & $0.598 \pm 0.429$ & 155 & 1.21 \\
\hline & $0.740 \pm 0.311$ & $0.487 \pm 0.007$ & $27.929 \pm 13.745$ & $(2.971 \pm 7.309) E 14$ & $0.254 \pm 0.302$ & - & - \\
\hline \multicolumn{8}{|l|}{$\overline{\mathrm{Pre}+\mathrm{SPs}}$} \\
\hline \multirow{2}{*}{$060502 \mathrm{~B}$} & $0.494 \pm 0.165$ & $-0.340 \pm 0.004$ & $-38.813 \pm 33.342$ & $(1.317 \pm 1.080) E 15$ & $0.700 \pm 0.182$ & 154 & 1.08 \\
\hline & $1.222 \pm 0.154$ & $0.015 \pm 0.003$ & $254.514 \pm 1745.588$ & 108.761 & $0.700 \pm 4.563$ & - & - \\
\hline \multirow[t]{2}{*}{ 071112B } & $0.438 \pm 1.325$ & $-0.568 \pm 0.005$ & $20.961 \pm 25.776$ & $(-1.424 \pm 1.410) E 15$ & $0.936 \pm 0.427$ & 204 & 1.04 \\
\hline & $0.403 \pm 0.067$ & $0.075 \pm 0.007$ & $26.953 \pm 8.395$ & $(-6.233 \pm 3.660) E 14$ & $1.012 \pm 0.305$ & - & - \\
\hline \multirow[t]{2}{*}{$100702 \mathrm{~A}$} & $0.548 \pm 0.231$ & $-0.250 \pm 0.004$ & $19.330 \pm 17.660$ & $(-7.487 \pm 7.021) E 14$ & $0.521 \pm 0.242$ & 116 & 0.93 \\
\hline & $1.482 \pm 0.101$ & $0.086 \pm 0.003$ & $-26.920 \pm 13.772$ & $(1.872 \pm 1.110) E 14$ & $1.241 \pm 0.728$ & - & - \\
\hline \multirow[t]{2}{*}{$\overline{160408 \mathrm{~A}}$} & $0.552 \pm 1.278$ & $-0.928 \pm 0.105$ & $79.970 \pm 956.924$ & 1.692 & $0.997 \pm 0.235$ & 316 & 1.06 \\
\hline & $0.646 \pm 0.106$ & $0.242 \pm 0.016$ & $94.775 \pm 22.713$ & $(-1.616 \pm 0.743) E 15$ & $12.686 \pm 3.267$ & - & - \\
\hline \multirow[t]{2}{*}{$160726 \mathrm{~A}$} & $1.474 \pm 0.564$ & $0.020 \pm 0.003$ & $-68.274 \pm 10.965$ & $(-4.360 \pm 1.310) E 15$ & $0.998 \pm 0.171$ & 242 & 1.14 \\
\hline & $1.616 \pm 0.151$ & $0.617 \pm 0.005$ & $-24.922 \pm 21.999$ & $(-7.269 \pm 11.494) E 14$ & $1.000 \pm 1.487$ & - & - \\
\hline \multirow[t]{2}{*}{$180402 \mathrm{~A}$} & $1.359 \pm 65.982$ & $-0.203 \pm 0.514$ & $463.348 \pm 61482.680$ & $25.266 \pm 1759.530$ & $0.488 \pm 21.032$ & 129 & 1.12 \\
\hline & $0.923 \pm 0.378$ & $0.195 \pm 0.009$ & $9.312 \pm 1.557$ & $(-7.793 \pm 1.723) E 14$ & $0.498 \pm 0.106$ & - & - \\
\hline$\overline{\text { Pre+Mt-D }}$ & & & & & & & \\
\hline & $0.448 \pm 3.709$ & $-0.376 \pm 0.040$ & 6.606 & $(1.017$ & 1.45 & 205 & 1.38 \\
\hline & $0.777 \pm 0.121$ & $0.048 \pm 0.008$ & $-201.869 \pm 199.456$ & $(-1.336 \pm 2.153) E 15$ & $-10.802 \pm 10.544$ & - & - \\
\hline & $1.058 \pm 0.086$ & $0.213 \pm 0.009$ & $(3.844 \pm 6.463) E 6$ & $(-6.166 \pm 15.290) E 18$ & $(2.648 \pm 4.395) E 5$ & - & 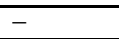 \\
\hline sGRE & & & & & & & \\
\hline$\overline{050724}$ & $1.256 \pm 0.123$ & $0.084 \pm 0.006$ & $(2.524 \pm 0.409) E 13$ & $(-1.043 \pm 0.809) E 26$ & $(-2.498 \pm 0.391) E 12$ & 266 & 1.20 \\
\hline & $0.280 \pm 0.055$ & $1.043 \pm 0.025$ & $338.931 \pm 37.597$ & $(7.507 \pm 0.424) E 16$ & $-40.226 \pm 4.399$ & - & - \\
\hline 130603B & $15.022 \pm 2.499$ & $0.017 \pm 0.004$ & $86.579 \pm 129.604$ & $5.348 \pm 4.760$ & $0.112 \pm 0.148$ & 174 & 1.17 \\
\hline & $4.828 \pm 0.832$ & $0.072 \pm 0.002$ & $16.574 \pm 226.295$ & $14.620 \pm 140.411$ & $0.108 \pm 2.183$ & 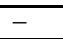 & - \\
\hline & $0.574 \pm 0.152$ & $0.192 \pm 0.010$ & $8.537 \pm 0.765$ & $(9.342 \pm 0.700) E 14$ & $0.384 \pm 0.041$ & - & - \\
\hline
\end{tabular}


Table 2. Characteristic Parameters of Diverse Gamma-ray Segments in sGRBs

\begin{tabular}{|c|c|c|c|c|c|c|c|c|c|c|c|c|c|c|c|c|c|}
\hline$\overline{G R B}$ & $\begin{array}{l}T_{90} \\
\mathrm{~s} \\
(2)\end{array}$ & $\begin{array}{c}\text { redshift } \\
\text { z } \\
(3)\end{array}$ & $\begin{array}{c}t_{\text {dur }} \\
\left(t_{s}, t_{e}\right) \\
(4)\end{array}$ & $\begin{array}{c}\text { Model } \\
\text { (5) }\end{array}$ & $\begin{array}{l}E_{p}{ }^{a} \\
k e V \\
(6)\end{array}$ & $\begin{array}{c}\text { Index } \\
\alpha \\
(7)\end{array}$ & $\begin{array}{c}\text { Flux }^{d} \\
(8)\end{array}$ & $\begin{array}{c}\text { Fluence }^{e} \\
\text { (9) }\end{array}$ & $\begin{array}{c}\chi^{2} / D O F \\
(10)\end{array}$ & $\begin{array}{c}\text { Model } \\
\text { (11) }\end{array}$ & $\begin{array}{c}E_{p} \\
k e V \\
(12)\end{array}$ & $\begin{array}{c}\text { Index } \\
\alpha \\
(13)\end{array}$ & $\begin{array}{c}\text { Flux d } \\
\text { (14) }\end{array}$ & $\begin{array}{c}\text { Fluence }^{e} \\
(15)\end{array}$ & $\begin{array}{c}\chi^{2} / D O F \\
(16)\end{array}$ & $\begin{array}{l}\Delta \chi^{2} \\
(17)\end{array}$ & $\begin{array}{c}\text { best-fit } \\
\text { Model } \\
(18)\end{array}$ \\
\hline \multicolumn{18}{|l|}{$\overline{\mathrm{SPs}}$} \\
\hline 070923 & 0.040 & - & $(-0.003,0.047)$ & $\mathrm{PL}$ & & $0.79 \pm 0.16^{c}$ & $24.90 \pm 14.42$ & $12.40 \pm 7.19$ & $66.02 / 73$ & CPL & $100.11 \pm 100.39$ & $0.17 \pm 0.63$ & - & - & $64.54 / 72$ & 1 & $\mathrm{PL}$ \\
\hline$\overline{090621 \mathrm{~B}}$ & 0.140 & - & $(0.011,0.111)$ & PL & & $0.73 \pm 0.15^{c}$ & $16.82 \pm 10.08$ & $16.85 \pm 10.10$ & $68.39 / 73$ & CPL & $135.29 \pm 156.51$ & $0.26 \pm 0.56$ & - & 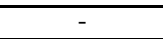 & $67.08 / 72$ & 1 & $\mathrm{PL}$ \\
\hline $100206 \mathrm{~A}$ & 0.116 & 0.4068 & $(-0.009,0.115)$ & PL & & $0.75 \pm 0.11^{c}$ & $29.85 \pm 19.52$ & $37.02 \pm 24.20$ & $72.02 / 73$ & CPL & $232.22 \pm 357.46$ & $0.49 \pm 0.40$ & - & - & $71.17 / 72$ & 1 & $\overline{\mathrm{PL}}$ \\
\hline 110420B & 0.084 & - & $(-0.000,0.052)$ & PL & - & $2.39 \pm 0.36$ & - & - & $121.77 / 73$ & CPL & $12.98 \pm 5.76$ & $-1.70 \pm 1.50$ & $3.34 \pm 1.97$ & $1.77 \pm 1.04$ & $99.41 / 72$ & 22 & CPL \\
\hline $120305 \mathrm{~A}$ & 0.100 & - & $(-0.005,0.122)$ & $\overline{\mathrm{PL}}$ & - & $1.13 \pm 0.06$ & - & - & $63.73 / 73$ & $\overline{\mathrm{CPL}}$ & $108.05 \pm 48.70$ & $0.61 \pm 0.24$ & $22.21 \pm 23.39$ & $28.09 \pm 29.58$ & $57.43 / 72$ & $(>) 6$ & $\overline{\mathrm{CPL}}$ \\
\hline $131004 \mathrm{~A}$ & 1.536 & 0.717 & $(-0.144,0.386)$ & PL & - & $1.77 \pm 0.07$ & - & - & $87.72 / 73$ & CPL & $51.57 \pm 19.01$ & $0.84 \pm 0.32$ & $3.31 \pm 3.24$ & $17.54 \pm 17.18$ & $77.77 / 72$ & 10 & CPL \\
\hline $140622 \mathrm{~A}$ & 0.13 & 0.959 & $(0.000,0.040)$ & PL & $51.17 \pm 2.71$ & $1.95 \pm 0.37$ & $4.18 \pm 2.06$ & $1.65 \pm 0.81$ & $46.13 / 73$ & CPL & $79.36 \pm 227.53$ & $1.42 \pm 1.59$ & & & $45.96 / 72$ & 0 & PL \\
\hline $150301 \mathrm{~A}$ & 0.484 & - & $(-0.002,0.051)$ & PL & - & $1.70 \pm 0.11$ & - & - & $77.69 / 73$ & CPL & $43.43 \pm 19.02$ & $0.55 \pm 0.49$ & $7.47 \pm 7.14$ & $3.95 \pm 3.77$ & $68.66 / 72$ & 9 & CPL \\
\hline 150710 & 0.152 & - & $(0.012,0.090)$ & PL & & $0.55 \pm 0.11^{c}$ & $29.20 \pm 18.51$ & $22.86 \pm 14.49$ & $87.41 / 73$ & CPL & $95.12 \pm 61.42$ & $-0.16 \pm 0.46$ & 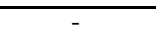 & & $83.77 / 72$ & 4 & PL \\
\hline $160601 \mathrm{~A}$ & 0.120 & - & $(-0.006,0.106)$ & PL & - & $1.47 \pm 0.13$ & - & - & $90.92 / 73$ & CPL & $75.20 \pm 43.70$ & $0.54 \pm 0.42$ & $5.58 \pm 5.68$ & $6.24 \pm 6.35$ & $78.43 / 72$ & 12 & $\overline{\mathrm{CPL}}$ \\
\hline $180727 \mathrm{~A}$ & 1.056 & - & $(0.205,0.771)$ & PL & $94.23 \pm 1.78$ & $1.65 \pm 0.18$ & $0.37 \pm 0.22$ & $2.09 \pm 1.24$ & $77.92 / 73$ & $\overline{\mathrm{CPL}}$ & $32.87 \pm 21.57$ & $0.22 \pm 0.88$ & - & & $72.39 / 72$ & $(<) 6$ & PL \\
\hline 190326A & 0.076 & - & $(-0.002,0.067)$ & PL & - & $2.66 \pm 0.19$ & - & - & $97.22 / 73$ & CPL & $24.85 \pm 14.61$ & $0.85 \pm 0.93$ & $7.79 \pm 6.61$ & $5.32 \pm 4.52$ & $86.02 / 72$ & 11 & $\mathrm{CPL}$ \\
\hline \multicolumn{18}{|l|}{ Mt-DPs } \\
\hline \multirow[t]{2}{*}{ 101219A } & 0.828 & 0.718 & $(-0.028,0.369)$ & $\mathrm{PL}$ & & $\mathbf{0 . 7 8} \pm 0.06^{c}$ & $9.12 \pm 6.27$ & $36.22 \pm 24.90$ & $63.97 / 73$ & CPL & $500.00 \pm 1559.14$ & $0.65 \pm 0.21$ & - & - & $64.23 / 72$ & 0 & $\overline{\mathrm{PL}}$ \\
\hline & & & $(0.369,0.622)$ & $\mathrm{PL}$ & $162.29 \pm 7.26$ & $1.42 \pm 0.56$ & $0.36 \pm 0.15$ & $0.90 \pm 0.37$ & $55.82 / 73$ & CPL & $213.82 \pm 1999.98$ & $1.15 \pm 2.09$ & - & - & $55.81 / 72$ & 0 & $\mathrm{PL}$ \\
\hline \multirow[t]{2}{*}{$120804 \mathrm{~A}$} & 0.808 & 1.3 & $(-0.126,0.194)$ & PL & $173.51 \pm 1.15$ & $1.39 \pm 0.08$ & $15.90 \pm 10.66$ & $50.82 \pm 34.08$ & $91.45 / 73$ & CPL & $133.82 \pm 93.43$ & $0.95 \pm 0.32$ & - & - & $89.15 / 72$ & 2 & $\mathrm{PL}$ \\
\hline & & & $(0.194,0.532)$ & PL & - & $2.31 \pm 0.06$ & - & - & $269.15 / 73$ & CPL & $24.87 \pm 2.63$ & $-0.70 \pm 0.26$ & $11.06 \pm 11.44$ & $37.45 \pm 38.74$ & $81.86 / 72$ & 187 & $\overline{\mathrm{CPL}}$ \\
\hline \multirow[t]{2}{*}{$130912 \mathrm{~A}$} & 0.284 & - & $(0.005,0.144)$ & PL & $73.51 \pm 1.38$ & $1.77 \pm 0.15$ & $7.87 \pm 4.85$ & $10.94 \pm 6.75$ & $71.36 / 73$ & CPL & $72.25 \pm 53.86$ & $0.94 \pm 0.54$ & - & - & $66.98 / 72$ & 4 & PL \\
\hline & & & $(0.144,0.299)$ & $\mathrm{PL}$ & & $0.74 \pm 0.19^{c}$ & $14.10 \pm 7.54$ & $21.84 \pm 11.68$ & $60.81 / 73$ & $\mathrm{CPL}$ & $487.09 \pm 4493.18$ & $0.59 \pm 0.69$ & - & - & $60.64 / 72$ & 0 & $\mathrm{PL}$ \\
\hline \multicolumn{18}{|l|}{$\overline{\text { Ml-DPs }}$} \\
\hline \multirow[t]{2}{*}{$120229 \mathrm{~A}$} & 0.22 & - & $(0.023,0.057)$ & $\mathrm{PL}$ & $104.58 \pm 3.43$ & $1.60 \pm 0.32$ & $2.83 \pm 1.40$ & $0.96 \pm 0.48$ & $80.75 / 73$ & CPL & $111.33 \pm 225.58$ & $1.01 \pm 1.17$ & - & - & $80.38 / 72$ & 0 & $\mathrm{PL}$ \\
\hline & & & $(0.178,0.242)$ & $\mathrm{PL}$ & & $0.63 \pm 0.62^{c}$ & $4.41 \pm 1.79$ & $2.83 \pm 1.14$ & $73.54 / 73$ & CPL & $27.17 \pm 49.45$ & $-1.26 \pm 3.48$ & - & - & $71.73 / 72$ & 2 & $\mathrm{PL}$ \\
\hline \multirow[t]{2}{*}{$111117 \mathrm{~A}$} & 0.464 & 2.211 & $(0.051,0.185)$ & PL & & $\mathbf{0 . 7 3} \pm \mathbf{0 . 2 1 ^ { c }}$ & $9.64 \pm 5.18$ & $12.92 \pm 6.94$ & $78.87 / 73$ & $\mathrm{CPL}$ & $139.07 \pm 313.14$ & $0.31 \pm 0.76$ & - & - & $77.09 / 72$ & 2 & $\mathrm{PL}$ \\
\hline & & & $(0.442,0.516)$ & $\mathrm{PL}$ & - & $1.23 \pm 0.20$ & - & - & $79.12 / 73$ & $\overline{\mathrm{CPL}}$ & $50.13 \pm 41.00$ & $0.00 \pm 0.79$ & $5.23 \pm 3.94$ & $3.87 \pm 2.91$ & $69.60 / 72$ & 10 & $\overline{\mathrm{CPL}}$ \\
\hline \multicolumn{18}{|l|}{ Pre+SPs } \\
\hline \multirow[t]{2}{*}{$060502 \mathrm{~B}$} & 0.144 & 0.287 & Pre:(-0.406,-0.391) & $\overline{\mathrm{PL}}$ & $67.50 \pm 3.22$ & $1.81 \pm 0.38$ & $1.17 \pm 0.58$ & $0.18 \pm 0.09$ & $67.83 / 73$ & $\mathrm{CPL}$ & $495.33 \pm 6729.11$ & $1.71 \pm 1.42$ & - & - & $67.98 / 72$ & 0 & $\overline{\mathrm{PL}}$ \\
\hline & & & Main:(0.004,0.073) & PL & - & $2.27 \pm 0.15$ & - & - & $110.66 / 73$ & CPL & $23.34 \pm 5.10$ & $-0.81 \pm 0.60$ & $2.54 \pm 2.49$ & $1.77 \pm 1.73$ & $70.09 / 72$ & 41 & CPL \\
\hline \multirow[t]{2}{*}{$071112 \mathrm{~B}$} & 0.304 & - & Pre:(-0.581,-0.557) & PL & $45.96 \pm 4.52$ & $2.01 \pm 0.65$ & $1.06 \pm 0.53$ & $0.25 \pm 0.13$ & $71.92 / 73$ & CPL & $16.28 \pm 15.35$ & $-1.49 \pm 3.11$ & - & - & $67.30 / 72$ & 5 & PL \\
\hline & & & Main:(0.050,0.094) & PL & $358.43 \pm 6.25$ & $1.14 \pm 0.33$ & $4.82 \pm 2.48$ & $2.14 \pm 1.10$ & $69.56 / 73$ & CPL & $38.59 \pm 42.46$ & $-0.54 \pm 1.57$ & - & - & $64.56 / 72$ & 5 & PL \\
\hline \multirow[t]{2}{*}{$100702 \mathrm{~A}$} & 0.512 & - & Pre:(-0.267,-0.238) & PL & & $2.97 \pm 0.81^{b}$ & $1.21 \pm 0.52$ & $0.35 \pm 0.15$ & $59.88 / 73$ & $\mathrm{CPL}$ & $5.94 \pm 9.16$ & $-1.97 \pm 7.33$ & - & - & $58.22 / 72$ & 2 & $\overline{\mathrm{PL}}$ \\
\hline & & & Main:(0.020,0.214) & PL & $112.22 \pm 1.05$ & $1.57 \pm 0.09$ & $9.91 \pm 6.78$ & $19.23 \pm 13.17$ & $75.79 / 73$ & $\mathrm{CPL}$ & $66.97 \pm 32.86$ & $0.76 \pm 0.40$ & - & - & $71.07 / 72$ & 5 & PL \\
\hline \multirow[t]{2}{*}{$160408 \mathrm{~A}$} & 0.320 & - & Pre:(-0.931,-0.910) & PL & $59.09 \pm 7.89$ & $1.88 \pm 1.00$ & $1.24 \pm 0.48$ & $0.26 \pm 0.10$ & $27.96 / 73$ & CPL & $10.13 \pm 69.94$ & $1.22 \pm 14.00$ & - & - & $28.84 / 72$ & 1 & $\mathrm{PL}$ \\
\hline & & & Main:(0.073,0.362) & $\mathrm{PL}$ & & $0.83 \pm 0.16^{c}$ & $13.01 \pm 7.28$ & $37.71 \pm 21.10$ & $52.75 / 73$ & $\mathrm{CPL}$ & $500.00 \pm 4036.87$ & $0.70 \pm 0.57$ & - & - & $52.99 / 72$ & 0 & $\mathrm{PL}$ \\
\hline \multirow[t]{2}{*}{$160726 \mathrm{~A}$} & 0.728 & - & Pre:(0.001,0.051) & PL & $231.93 \pm 2.93$ & $1.29 \pm 0.19$ & $11.70 \pm 6.75$ & $5.83 \pm 3.37$ & $66.58 / 73$ & $\mathrm{CPL}$ & $54.8 \pm 41.53$ & $0.14 \pm 0.80$ & - & - & $62.61 / 72$ & 4 & $\mathrm{PL}$ \\
\hline & & & Main:(0.535,0.766) & $\mathrm{PL}$ & - & $1.37 \pm 0.08$ & - & - & $84.00 / 73$ & CPL & $61.39 \pm 17.32$ & $0.23 \pm 0.30$ & $10.08 \pm 9.99$ & $23.30 \pm 23.08$ & $61.75 / 72$ & 22 & $\mathrm{CPL}$ \\
\hline \multirow[t]{2}{*}{$180402 \mathrm{~A}$} & 0.180 & - & Pre:(-0.205,-0.194) & PL & $62.71 \pm 3.12$ & $1.85 \pm 0.38$ & $1.40 \pm 0.91$ & $0.16 \pm 0.10$ & $78.22 / 73$ & $\mathrm{CPL}$ & $72.98 \pm 130.89$ & $1.09 \pm 1.40$ & - & - & $77.36 / 72$ & 1 & PL \\
\hline & & & Main:(0.135,0.250) & PL & - & $0.47 \pm 0.22$ & - & - & $147.13 / 73$ & $\mathrm{CPL}$ & $81.52 \pm 81.74$ & $-0.41 \pm 0.91$ & $13.72 \pm 9.19$ & $15.73 \pm 10.54$ & $68.46 / 72$ & 79 & CPL \\
\hline \multicolumn{18}{|c|}{ 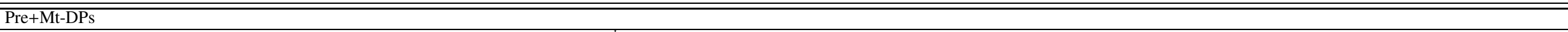 } \\
\hline $100625 \mathrm{~A}$ & 0.332 & 0.452 & Pre:(-0.384,-0.370) & $\mathrm{PL}$ & & $2.80 \pm 1.72^{b}$ & $0.16 \pm 0.04$ & $0.02 \pm 0.01$ & $37.91 / 73$ & CPL & $14.63 \pm 58.38$ & $0.00 \pm 9.95$ & & & $36.98 / 72$ & 1 & $\mathrm{PL}$ \\
\hline & & & Main1:(-0.059,0.141) & PL & - & $0.99 \pm 0.09$ & - & - & $87.56 / 73$ & CPL & $63.73 \pm 39.92$ & $0.07 \pm 0.38$ & $7.59 \pm 7.39$ & $15.19 \pm 14.78$ & $78.09 / 72$ & 9 & CPL \\
\hline & & & Main2:(0.141,0.262) & PL & & $1.01 \pm 0.10^{c}$ & $16.51 \pm 10.87$ & $19.98 \pm 13.16$ & $95.51 / 73$ & CPL & $127.76 \pm 108.59$ & $0.56 \pm 0.38$ & - & - & $93.12 / 72$ & 2 & $\mathrm{PL}$ \\
\hline$\overline{s G R B s+1}$ & & & & & & & & & & & & & & & & & \\
\hline 050724 & 98.684 & 0.257 & Main:(-0.020,0.243) & PL & $167.65 \pm 1.19$ & $1.41 \pm 0.08$ & $12.50 \pm 8.42$ & $32.92 \pm 22.18$ & $80.55 / 73$ & $\overline{\mathrm{CPL}}$ & $179.46 \pm 185.48$ & $1.10 \pm 0.33$ & - & - & $79.50 / 72$ & 1 & PL \\
\hline & & & EE:(0.922,1.247) & PL & $52.64 \pm 2.15$ & $1.94 \pm 0.29$ & $1.56 \pm 0.79$ & $5.08 \pm 2.58$ & $62.77 / 73$ & $\overline{\mathrm{CPL}}$ & $120.22 \pm 329.47$ & $1.56 \pm 1.15$ & - & - & $62.62 / 72$ & 0 & PL \\
\hline $130603 \mathrm{~B}$ & 0.176 & 0.3565 & Main1:(-0.008,0.051) & PL & & $0.62 \pm 0.06^{c}$ & $169.00 \pm 116.73$ & $99.24 \pm 68.55$ & $90.12 / 73$ & $\mathrm{CPL}$ & $196.17 \pm 142.43$ & $0.31 \pm 0.21$ & - & - & $87.48 / 72$ & 3 & $\mathrm{PL}$ \\
\hline & & & Main2:(0.051,0.162) & $\mathrm{PL}$ & & $0.81 \pm 0.05^{c}$ & $74.00 \pm 51.20$ & $82.44 \pm 57.04$ & $70.41 / 73$ & CPL & $453.76 \pm 648.62$ & $0.68 \pm 0.19$ & - & - & $69.51 / 72$ & 1 & $\mathrm{PL}$ \\
\hline & & & EE:(0.162,0.260) & $\mathrm{PL}$ & $33.40 \pm 0.93$ & $2.20 \pm 0.15$ & $3.61 \pm 2.27$ & $3.52 \pm 2.21$ & $79.50 / 73$ & $\overline{\mathrm{CPL}}$ & $27.23 \pm 16.03$ & $0.75 \pm 0.82$ & - & - & $74.52 / 72$ & 5 & PL \\
\hline
\end{tabular}

${ }^{a} E_{p}$ estimated using Eq. 5 for the segments whose best-fit model is PL with $1.2 \leq \alpha \leq 2.3$ and marked in italics (Zhang et al. 2007a). ${ }^{b}$ The segments whose best-fit model is PL with $\alpha>2.3$ marked in boldface. ${ }^{c}$ The segments whose best-fit model is PL with $\alpha<1.2$ marked in boldface. ${ }^{d} F_{\text {lux }}$ of the best-fit model in unit of $10^{-7} \mathrm{ergcm}^{-2} \mathrm{~s}^{-1}$. ${ }^{e} S_{\gamma}$ of the best-fit model in unit of $10^{-8} \mathrm{ergcm}^{-2}$. 